\title{
Biological Mechanisms Underlying Addiction
}

\author{
Ilhan Yargic
}

\begin{abstract}
$\underline{\text { Abstract }}$
Addiction is a behavioral disorder related to alterations in neurobiological systems involved in reward system, brain stress response, physical withdrawal, inhibition and executive control. Alcohol or drug addiction does not occur without using these substances but genetic and epigenetic variations in these neurobiological systems cause individual differences. The current review summarizes the literature on the biological basis of drug addiction. In addition, this review tries to explain the path from occasional recreational substance use to the compulsive, addicted state. It will help understand why avoiding psychoactive drugs or not to start using is very crucial.
\end{abstract}

Keywords: addiction, mechanism, abuse, dependence, neurobiology

International Journal of Human and Health Sciences Vol. 02 No. 03 July'18 Page : 107-111 DOI: http://dx.doi.org/10.31344/ijhhs.v2i3.37

\section{Introduction}

Drug addiction is a chronic, relapsing disorder that is characterized by a craving for drug, compulsive drug use, loss of control in limiting intake; and emergence of an aversive state (withdrawal) when drug is not accessed ${ }^{1}$. Although any drug use has a potential for further abuse or dependence, clinical experience and animal studies show that neurobiological effects of occasional and limited use of a drug, like alcohol, is different from those of a chronic dependent state ${ }^{2}$. Addiction develops as a result of a transition from the first neurobiological state to the second. Drug intake, accompanied by a biological vulnerability causes some permanent functional and structural changes ${ }^{3}$ in various parts of the central nervous system (CNS) and several neuroendocrinological modulator systems.

While talking about the health hazards of alcohol and drugs, usually their deteriorative effects on bodily functions and organs like liver and lung are mentioned first. However, maybe the worst effect of alcohol and drugs is on brain, because they cause long term neuroadaptive changes in the CNS which lead to compulsive drug use despite bad consequences and relapse even long after acute withdrawal symptoms abate. Modern research on drug abuse has demonstrated the biochemical, cellular, genetic, epigenetic and circuitry mechanisms that mediate the progression from experimenting with a drug to addiction.

Addiction is a brain disease that starts with drug use. It is not just a matter of will power. A person who uses a drug is taking the risk of starting this disease process in his body and brain because drug use triggers a series of biological cascades. These changes in the brain have a potential to continue for a long time after drug use has stopped. Addiction is a biopsychosocial disorder similar to other chronic physiological disorders such as diabetes mellitus that are progressive and influenced by environmental factors and stressors. A diabetic person cannot claim that he will use his will power to keep a normal blood glucose level after he consumes a large amount of carbohydrate. Just like that, an addict cannot keep limiting his drug use and avoid bad consequences forever. But like a diabetic person who should avoid food that is rich from carbohydrates, an addict should take every precaution to stay away from drugs for good.

Alcohol and drugs have effects on four interrelated biological systems in the brain that underlie addiction. These are brain reward system, brain stress systems, autonomic nervous system (related to physical withdrawal) and prefrontal cortex function (related to cognitive inhibitory control). Drug use produces biological changes that ruin the homeostatic operation of the CNS and a new

Correspondence to: Prof. Dr. Ilhan Yargic, M.D. (Psychiatrist) Private Practice, Istanbul, Turkey E-mail: iyargic@hotmail.com 
state of chronic dysregulation (an allostatic state) is shaped ${ }^{4}$.

\section{Brain Reward Systems}

Reward system is the fuel of life. Mesolimbic dopaminergic system that connects nucleus accumbens and ventero-tegmental area and gives projections to the prefrontal cortex is the neural structure of the reward pathway. This brain circuit is present in human and all animals and it gives an appetitive stimulus to obtain innate needs (food and sex) that are necessary for survival of the individual and continuation of the generations. The pleasure imbedded in them is like a small fee given to the creatures to turn the wheel of physical existence. Through this mechanism, Allah has programmed us to want things that we need to survive. For example, eating food is not a burden that we just have to do but it is a pleasure we seek for. Rewards serve as positive reinforcers because they affect the behavior towards obtaining a goal that results in pleasure and satisfaction. When someone uses a psychoactive drug, he is abusing the reward system outside its special purpose. Almost all drugs of abuse stimulate mesolimbic dopaminergic system much stronger than the natural stimuli, thus they produce pleasurable effects initially. Repetitive stimulation of this system causes sensitization that is responsible of craving for drug. Drugs act like computer viruses that change the original program of a computer (the brain) and reorganize the system to perceive them as a basic need, re-program the system to produce an urge for them (drugs). Drug user imagines this strong desire for drug as his natural will. Especially in the initial phases of addiction, they say "I use it as I want to use it". They do not take into consideration that this urge is not innate and it develops after drug use. This strong feeling of desire (impulse) for drug use that affects decision-making process of the person is one of the key elements of addiction.

Most of the drugs of abuse increase dopamine in the mesolimbic system directly or indirectly to produce a reward or a high. For example, opiates act on opioid receptors which than stimulate dopamine receptors; cocaine competes with dopamine in the synaptic cleft for the dopamine reuptake pump and keeps dopamine in the cleft. Activation of dopamine neurons is a motivational factor that produces reward-anticipation. When a reward is higher than expected, sensitization of dopamine neurons increases. This sensitization increases the urge or motivation for the reward ${ }^{5}$.
Drugs may produce differenteffects on individuals ${ }^{6}$. For example, cocaine or methylphenidate use increases dopamine levels in human brain and this increase is associated with pleasure. If drug-naïve subjects had low levels of dopamine $\mathrm{D}_{2}$ receptors, they experienced pleasure. But if they originally had high receptor levels, they experienced unpleasant feelings. This study explains the biological mechanism of why drug use is pleasurable for some people and tends to be repetitive while it is unpleasant and not repetitive for other ${ }^{6}$.

Neurotransmitters other than dopamine also play a role in reward system ${ }^{7}$. Dopamine independent reward mechanisms have been described for opioids and alcohol. Serotonergic receptors in the nucleus accumbens mediate the reinforcing effects of psychostimulant drugs. Stimulation of $\mu$-opioid receptors in the nucleus accumbens and ventral tegmental area are responsible for the reinforcing effects of opioids. Opioid receptors in the ventral striatum and amygdala mediate the reinforcing effects of ethanol. Acute intoxicating doses of alcohol also increase inhibitory $\gamma$-aminobutyric acid (GABA) in the amygdala.

Activation of the mesolimbic dopamine system produces an incentive salience linked to the environmental stimuli and drives goal-directed behavior ${ }^{1}$. Pleasure (hedonic state) provided by the reward starts a learning process including conditioning with cues that are associated with drug use, assigning value and motivational status to the reward. Motivational state produced by drug withdrawal is like hunger and sexual arousal that increase the incentive salience of the reward and related cues ${ }^{8}$. As the hunger or withdrawal rises, struggle to get the reward will accelerate.

Brain has a plastic property and it rapidly develops an adaptation to the effects of drugs. This is called tolerance. Repetitive use of a drug increases reward thresholds (decreases reward). For this reason, drug use does not produce satiety as in natural rewards like food. A person can eat up to a certain amount of his favorite food each time but increases the amount of a drug in subsequent uses. The person cannot get the same high from a drug during chronic use but he still experiences a high level of urge to use it. In other words, tolerance does not extinguish this desire. The brain keeps the value of possible actions in memory according to the amount of reward it produced in the past ${ }^{5}$. This stored value is used to evaluate possible results of future actions as reward or punishment. 


\section{Brain Stress Systems}

Stress system is the brain alarm circuit that is triggered by danger or unpleasant stimuli. Some neurochemicals that are activated by acute or chronic stressors initiate some typical behavioral responses. In animals various behavioral responses like freezing or flight may be observed ${ }^{1}$. Brain stress systems include neurocircuitry mediated by glucocorticoids, corticotropin-releasing factor (CRF), norepinephrine, and dynorphin. There are also neurochemicals like neuropeptide Y (NPY), nociceptin, and endocannabinoids that oppose the brain stress systems.

Stimulation of nucleus accumbens, the reward system, also activates brain stress systems that subsequently feedback to decrease dopamine release in the mesolimbic dopamine system. Brain stress systems aim to neutralize the effects of the drug and restore normal function despite the presence of drug. This causes tolerance to the effects of the drug use and dysphoric syndrome when the use is stopped because the change in stress systems continues although the drug use is avoided ${ }^{1}$.

Adrenocorticotropic hormone (ACTH) and corticosterone are elevated during withdrawal and this disturbs the hypothalamic-pituitaryadrenal (HPA) axis and extrahypothalamic brain stress system mediated by CRF. Withdrawal and protracted abstinence from drugs produce anxiety and irritability mediated by $\mathrm{CRF}^{1}$. Dynorphin activation mediates depressive responses to stress and dysphoric responses during withdrawal from drug9.

Animal studies ${ }^{10}$ show that when primates were conditioned to associate a cue with a pleasurable object like food, increased dopaminergic activity was a response to the cue and not to the food. Absence of food caused a drop in dopaminergic function. Reduction in the dopaminergic activity is considered to be associated with negative affect like dysphoria. So when an addict comes across with a cue (e.g. needle, money), and cannot access the drug he may feel dysphoric. This negative feeling will also increase the drive to get the drug.

\section{Physical Withdrawal}

Withdrawal is the emergence of specific behavioral and physical symptoms following sudden discontinuation of a drug in subjects who had been under its chronic influence. It is the result of reversal of homeostatic mechanisms which had been disturbed by the drug before discontinuation ${ }^{11}$. In other words, withdrawal is the result of sudden backward change of the homeostatic mechanisms that caused tolerance to the drug. Actually in stimulants and heroin, tolerance starts to develop very early on and the person cannot get the same effect from the same amount of drug and needs progressively higher drug doses to get the same effect, so that the person gets into the vicious cycle of tolerance.

Acute physical withdrawal is related to the somatic effects of drug use. Long term use of many drugs leads to adaptations within the autonomic nervous system and its target organs. This adaptation is disturbed when drug use is avoided ${ }^{12}$. For this reason, medications that modulate the autonomic nervous system, such as clonidine and propranolol are effective at reducing acute withdrawal symptoms. Acute somatic symptoms of withdrawal abate in days to weeks after the drug is discontinued; however, a chronic or protracted withdrawal state, where the patient suffers from unpleasant psychological symptoms especially triggered by drug cues, lasts for months to years. Permanent changes in the brain may induce relapses to drug abuse long after detoxification.

Gamma-aminobutyric acid (GABA)-ergic system and the glutamatergic system play important role in alcohol withdrawal ${ }^{13}$. Increased glutamatergic NMDA function is involved in seizures and cell death. Amygdala and hippocampus are critical sites for glutamatergic hyperactivity.

Reduction in dopamine plays role in both early abstinence and protracted withdrawal from many drugs of abuse ${ }^{14}$. Neuroimaging studies have shown reduced dopaminergic activity in opiate, cocaine and alcohol addictions. Even partial recovery of this change takes several months ${ }^{6}$. Altered reward neurotransmitters increase brain reward thresholds (a higher set point for drug reward) during abstinence. This in turn produces the negative motivational state in withdrawal (psychological symptoms of withdrawal) and makes the patient vulnerable to relapse ${ }^{1}$. Withdrawal symptoms are aversive for the drug user; therefore, he starts to seek the drug not for pleasure but to avoid withdrawal symptoms.

\section{Impulse control and decision making}

Inhibitory control and decision making are key executive functions for the development of addiction and they are mediated by the forebrain. Prefrontal cortex (PFC) is involved in regulation of both limbic reward regions and higher-order executive functions. Therefore, in addiction, PFC dysfunction is not only related to compulsive 
drug use but also underlines the dysfunctional behavioral pattern of drug addicts. Impairment of the PFC functions is also related to salience attribution in addiction. "Salience attribution" is ascribing excessive prominence to the drug and drug related cues, decline in sensitivity to non-drug reinforcers and less ability to inhibit maladaptive behaviors ${ }^{15}$. This impairment makes drug seeking and drug use the main motivational drive even though it leads to long term losses. It leads to neglecting other activities and the person can engage in extreme behaviors in order to obtain drugs $^{16}$. The process ends in the weakening of free will.

Administration of drugs of dependence to drug naïve laboratory animals produces changes in PFC similar to those in human drug addicts. PFC impairment seen in drug addicts is a result of drug use that enables further use. However, PFC impairment is also present in several other psychiatric and neurological conditions such as borderline personality disorder, attention deficit and hyperactivity disorder (ADHD), schizophrenia and bipolar disorder and traumatic brain injury ${ }^{17}$. The presence of PFC impairment makes those patient groups more vulnerable to drug use and dependence.

Executive functions mediated by the prefrontal cortex are gained through biological maturation of the brain during adolescence. Therefore, adolescent brain is more vulnerable to the harmful effects of drug abuse ${ }^{18}$. Different parts of the $\mathrm{PFC}$ are related to distinct features of addiction; for example, medial orbitofrontal cortex and ventromedial prefrontal cortex to craving, orbitofrontal cortex to drug expectation, anterior cingulate cortex to attention bias and dorsolateral prefrontal cortex to drug-related memories ${ }^{15}$. Glutamatergic pathway starting from the PFC and controlling the dopaminergic neurons in the nucleus accumbens are associated to addiction. ${ }^{19}$. Several other neurotransmitter systems such as endogenous opioid, serotonergic, cannabinoid and dopaminergic systems are also involved in prefrontal impairment ${ }^{15}$.

Inhibitory control enables to take appropriate actions to accomplish complex tasks and provides adaptation to new environmental conditions by suppressing immediate or habitual responses. Impairment in the inhibitory control is a key element in repetitive substance misuse and dependence ${ }^{17}$. Deficit in impulse control can be demonstrated with neurocognitive tests like color word Stroop Task, continuous performance test and the stopsignal task. In laboratory gambling task where risky decision making is accessed, drug addicts tend to take actions associated with short-term gains although they can bring long-term losses ${ }^{17}$. The individuals with impulsive personality traits are more prone to try and be addicted to drugs and drug use puts these individuals into a vicious cycle of further impulsivity ${ }^{20}$.

Impairment in impulse control leads to a weakening in self control (the ability to postpone or avoid an activity that may not be appropriate or is perceived as the incorrect) $)^{21}$. This explains the inability of drug addicts to inhibit excessive drug use although they are aware of the destructive consequences. Impairment in impulse control and self control also underline engagement in criminal activities and aggression. Young individuals who already have weak self-control are more prone to substance dependence ${ }^{21}$.

\section{Conclusion}

Addicted brain is in state of chronic dysregulation (allostasis) where function of reward circuits are impaired and stress systems are activated both of which lead to increased impulses to use drugs. Somatic withdrawal symptoms as well as emotional dysregulation also increase the urge of drug use. On top of these, dysregulation of the frontal cortex which is supposed execute thoughts, impulses and emotions results in compulsive drug seeking and loss of control over intake. This is a process initiated by drug use and can be avoided primarily by staying away from drugs. 
International Journal of Human and Health Sciences Vol. 02 No. 03 July'18

\section{$\underline{\text { References }}$}

1. Koob GF. Addiction is a Reward Deficit and Stress Surfeit Disorder. Front Psychiatry. 2013; 4:72.

2. Koob GF. Theoretical Frameworks and Mechanistic Aspects of Alcohol Addiction: Alcohol Addiction as a Reward Deficit Disorder. Curr Top Behav Neurosci. $2013 ; 13: 3-30$.

3. Denier N, Schmidt A, Gerber H, et al. Association of frontal gray matter volume and cerebral perfusion in heroin addiction: a multimodal neuroimaging study. Front Psychiatry. 2013; 4:135.

4. Edwards S, Koob GF. Neurobiology of dysregulated motivational systems in drug addiction. Future Neurol. 2010; 5:393-401.

5. Arias-Carrión O, Pŏppel E. Dopamine, learning, and reward-seeking behavior. Acta Neurobiol Exp (Wars). 2007; 67:481-488.

6. Volkow ND, Fowler JS, Wang GJ. Imaging studies on the role of dopamine in cocaine reinforcement and addiction in humans. J Psychopharmacol. 1999; 13:337-345.

7. Nestler EJ. Is there a common molecular pathway for addiction? Nat Neurosci. 2005; 8:1445-1449.

8. Karoly HC, Harlaar N, Hutchison KE. Substance use disorders: a theory-driven approach to the integration of genetics and neuroimaging. Ann N Y Acad Sci. 2013; 1282:71-91.

9. Chartoff E, Sawyer A, Rachlin A, et al. Blockade of kappa opioid receptors attenuates the development of depressive-like behaviors induced by cocaine withdrawal in rats. Neuropharmacology 2012; 62:1167-1176

10. Schultz W. Reward signaling by dopamine neurons. Neuroscientist. 2001; 7:293-302.

11. Vetulani J. Drug addiction. Part II. Neurobiology of addiction. Pol J Pharmacol. 2001; 53:303-317.
12. Naqvi NH, Bechara A. The insula and drug addiction: an interoceptive view of pleasure, urges, and decisionmaking. Brain Struct Funct. 2010; 214:435-450

13. Roberto M, Gilpin NW, Siggins GR. The central amygdala and alcohol: role of $\gamma$-aminobutyric acid, glutamate, and neuropeptides. Cold Spring Harb Perspect Med. 2012; 2:a012195.

14. Murphy A, Taylor E, Elliott R. The detrimental effects of emotional process dysregulation on decision-making in substance dependence. Front Integr Neurosci. 2012; 6:101

15. Goldstein RZ, Volkow ND. Dysfunction of the prefrontal cortex in addiction: neuroimaging findings and clinical implications. Nat Rev Neurosci. 2011; 12:652-669.

16. Volkow ND, Li TK. Drug addiction: the neurobiology of behaviour gone awry. Nat Rev Neurosci. 2004; 5:963-970.

17. Li CS, Sinha R. Inhibitory control and emotional stress regulation: neuroimaging evidence for frontallimbic dysfunction in psycho-stimulant addiction. Neurosci Biobehav Rev. 2008; 32:581-597.

18. Selemon LD. A role for synaptic plasticity in the adolescent development of executive function. Transl Psychiatry. 2013; 3:e238

19. Lingford-Hughes A, Nutt D. Neurobiology of addiction and implications for treatment. $\mathrm{Br} \mathrm{J}$ Psychiatry. 2003; 182:97-100.

20. Dawe S, Loxton NJ. The role of impulsivity in the development of substance use and eating disorders. Neurosci Biobehav Rev. 2004; 28:343-351.

21. Moffitt TE, Arseneault L, Belsky D, et al. A gradient of childhood self-control predicts health, wealth, and public safety. Proc Natl Acad Sci U S A. 2011; 108:2693-2698. 